These findings are important because unlike other treatments reported in the NOD mouse this therapy is effective when administered relatively late in disease progression. Interestingly, NKG2D has been detected on T cells — isolated from the synovial tissue of rheumatoid arthritis patients - in the presence of one of its ligands, which raises the posibility that this receptor could play a detrimental role in other autoimmune diseases.

Melanie Brazil

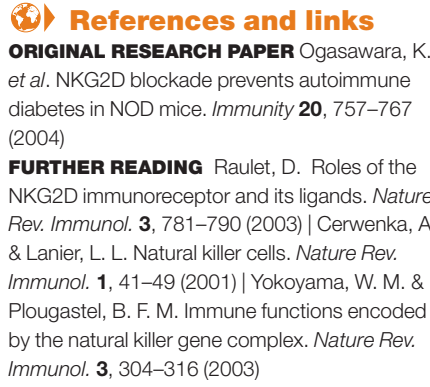

Immunol. 3, 304-316 (2003)

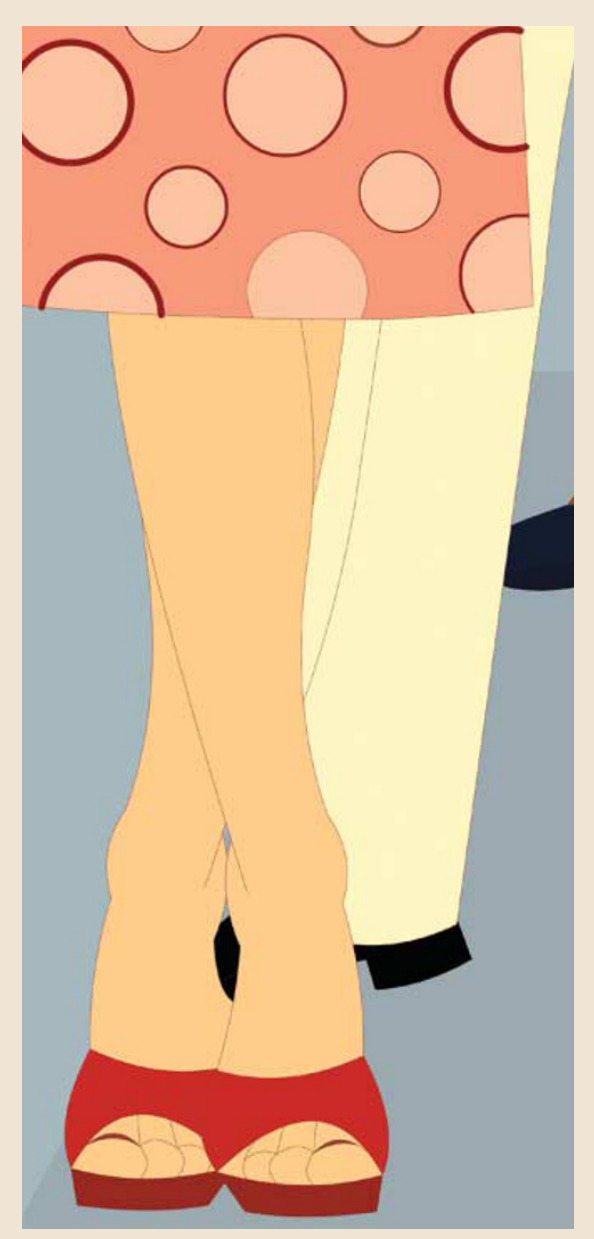

\section{Growing concern for tamoxifen}

Breast tumours that recur after treatment with the anti-oestrogen tamoxifen might result from a change in the effect of the drug that turns it from an antagonist of the oestrogen receptor into an agonist that promotes cancer cell growth. Writing in Cancer Cell, Rob Michalides and colleagues report that phosphorylation of oestrogen receptor- $\alpha$ (ER- $\alpha$ ) by protein kinase A (PKA) allows tamoxifen to bind but obliterates its antagonistic effect. Their findings mean that it will be possible to screen patients for activated PKA, which will indicate whether they would be more successfully treated with tamoxifen or a pure anti-oestrogen such as ICI-182780 (Fulvestrant/Faslodex).

Tamoxifen is a very effective treatment for oestrogen-receptor-positive $(\mathrm{ER}+)$ breast cancer that has few side effects and even beneficial properties such as promoting bone growth. However, $50 \%$ of recurrent ER+ breast cancers are resistant to tamoxifen. One of the proposed mechanisms for this resistance is phosphorylation of ER- $\alpha$ by PKA. Anti-oestrogens such as tamoxifen and ICI- 182780 act by binding to ER- $\alpha$ and causing a conformational change that prevents the recruitment of co-activators to ER- $\alpha$.

A recombinant ER- $\alpha$ was constructed that contained a different variant of green fluorescent protein at the amino and carboxyl termini to measure changes in the fluorescence resonance energy transfer (FRET) signal that occurs when the relative position of each fluorophore is altered by a change in receptor conformation. Using this assay, the authors studied the effect on receptor conformation of several factors that induce resistance to tamoxifen (SRC1, cAMP and cyclin D1).

FRET analysis showed that CAMP prevented tamoxifen from causing a conformational change of ER- $\alpha$, whereas SRC1 and cyclin D1 had no effect with or without cAMP. Not only did cAMP prevent tamoxifen antagonist action, it also caused the antioestrogen to behave as an agonist and upregulated ER- $\alpha$-responsive reporter genes. By contrast, cAMP could not overcome ICI-182780 antagonism of ER- $\alpha$. This is related to the fact that tamoxifen is a selective oestrogen receptor modulator (SERM) that behaves as either an agonist or antagonist depending on the cellular context, whereas ICI-182780 is a full antagonist.

The authors then investigated the mechanism of cAMP-induced resistance by studying the FRET pattern after exposure to forskolin, which stabilizes the generation of CAMP and therefore the activation of PKA. Forskolin prevented tamoxifen-induced

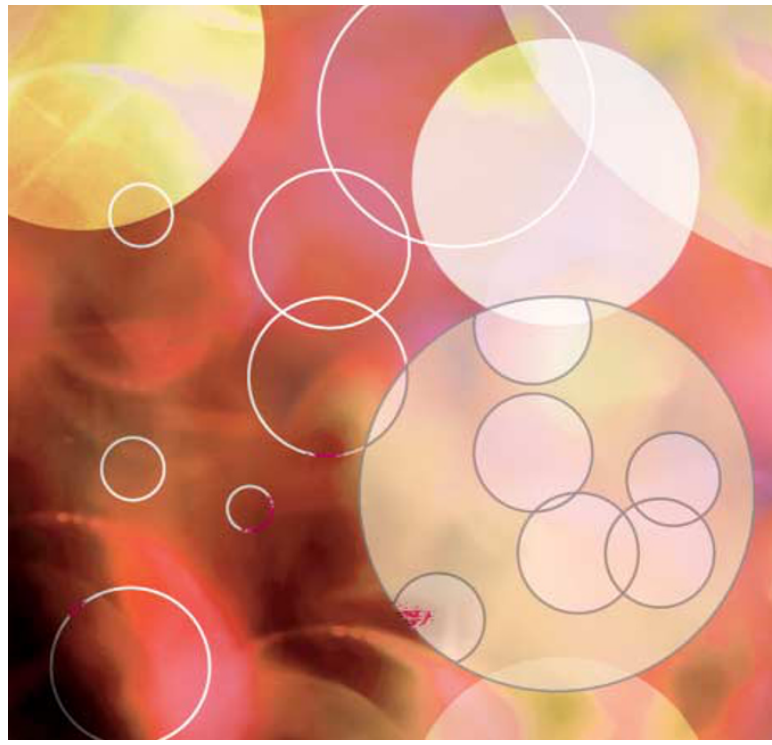

conformational change but had no effect on ICI182780. Even when a tenfold molar excess of ICI-182780 was added after tamoxifen treatment, no change in conformation was observed, which demonstrates that tamoxifen still binds to ER- $\alpha$ but is unable to induce the inhibitory conformation.

Mutational analysis of consensus PKA phosphorylation sites in ER- $\alpha$ showed that Ser305, which is located in the hinge region, is necessary for forskolin to prevent tamoxifen-induced conformational change, and indicates that PKA renders ER- $\alpha$ resistant to antagonism by tamoxifen.

To further dissect the role of the PKA pathway in tamoxifen resistance, the authors studied 70 $\mathrm{ER}+$ breast cancers isolated before tamoxifen treatment, 20 of which were later classed as tamoxifen-resistant. Gene expression analysis showed that tamoxifen-resistant breast tumours have downregulated PKA-RI- $\alpha$ (the PKA negative regulatory subunit). Correspondingly, RNA interference knockdown of PKA-RI- $\alpha$ induced ER- $\alpha$ resistance to tamoxifen, but not ICI-182780. Furthermore, T47D cells, which depend on oestrogen for growth, continued to grow in the presence of tamoxifen when PKA-RI- $\alpha$ was downregulated, whereas ICI-182780 retained its inhibitory effect on growth. This agonist action of tamoxifen induced by PKA explains the regression of tumours observed in tamoxifen-resistant patients when tamoxifen treatment is withdrawn. Selection of patients for anti-oestrogen treatment according to PKA activity should improve the success rate of tamoxifen treatment and prevent the further outgrowth of resistant tumour cells.

Joanna Owens

6) References and links ORIGINAL RESEARCH PAPER Michalides, R. et al. Tamoxifen resistance by a conformational arrest of the estrogen receptor $\alpha$ after PKA activation in breast cancer. Cancer Cell 5, 597-605 (2004) WEB SITE

Rob Michalides Lab: http://www.nki.nl/nkidep/h4/michalides/ michalides.htm 